\title{
MORGEN, DAS 22. JAHRHUNDERT Neue, alte und Andere Zukünfte
}

\author{
von VERA MADER
}

Armen Avanessian, Mahan Moalemi (Hg.):

Ethnofuturismen, Leipzig (Merve) 2018

Alexis Lothian: Old Futures. Speculative Fiction and Queer Possibility, New York (NYU Press) 2019

Henriette Gunkel, kara lynch (Hg.): We Travel the Space Ways. Black Imaginations, Fragments, and Diffractions, Bielefeld (transcript) 2019

Zukunft ist ungleich verteilt. Ob jemand von einem gesicherten Auskommen für sich und nächste Angehörige ausgehen kann, richtet sich nach der color line, der sozialen Herkunft und/oder der geschlechtlichen Identität. Wenn die Zukunft kid stuff ist, wie José Esteban Muñoz Lee Edelmans Inanspruchnahme von Queerness als Absage an eine (reproduktive) Zukunft entgegenhält, dann ist sie nur die Angelegenheit mancher Kids, ${ }^{1}$ nämlich jener, denen qua sozialer Positionierung eine solche vorbestimmt ist. Der Begriff der Zukunft, mit dem ich diese Rezension überschreibe, ist eine Erfindung und sinngebende Fiktion der weißen Moderne, ${ }^{2}$ die den nach wie vor konkreten Zusammenhang von race und Überleben vernachlässigt. Diese ‘Zukunft` posiert als Deutungshoheit, die jede Vision Anderer Zukünfte zu korrumpieren scheint. Ausgehend von prekären Lebensrealitäten, in der die Rassifizierung Schwarzer und Brauner Körper ¿Zukunft haben` zum Privileg weniger macht, verortete die Sängerin Nina Simone im Jahr
1971 die Möglichkeit Schwarzer Zukünftigkeit in einem Stadium nach dem apokalyptischen Zerfall: «When life is taken and there are no more babies born/[...]/Tomorrow will be the 22nd Centuryn. ${ }^{3}$ Wenn diese Versicherung des Kommenden auch von einer unter den gegebenen Bedingungen nicht verfügbaren Zukunft ausgeht, so ist in ihrer Formulierung sowohl der heilende Bruch eines Neubeginns als auch die zeitliche Kontinuität repressiver Strukturen angelegt.

Simones 22. Jahrhundert ist insofern Gegenstand dieser Rezension, als diese drei Veröffentlichungen vorstellt, die sich mit fiktionalen, künstlerischen und spekulativen Rekalibrierungen einer zivilisatorischen ¿Zukunft befassen - als einer wirkmächtigen Denkfigur der Moderne, deren Versprechen entlang sozialer Differenzachsen ihre Gültigkeit verlieren. Die Bände Ethnofuturismen, herausgegeben von Armen Avanessian und Mahan Moalemi, und We Travel the Space Ways. Black Imaginations, Fragments, and Diffractions, erschienen in Ko-Herausgeber_innenschaft von Henriette Gunkel und kara lynch, sowie die Monografie Old Futures. Speculative Fiction and Queer Possibility von Alexis Lothian denken Zukunft von sozialen Positionierungen jenseits des aufklärerischen Subjekts aus. In der Regel werden historisch marginalisierte Personengruppen nicht in eine Zukunft miteinbezogen, die mit dem Determinismus von Moderne/Kolonialität ${ }^{4}$ verschweißt und dabei ebenso zeitlich linear wie straight in dem Sinne ist, dass deren soziale Reproduktion normativ organisiert ist. Demgegenüber 


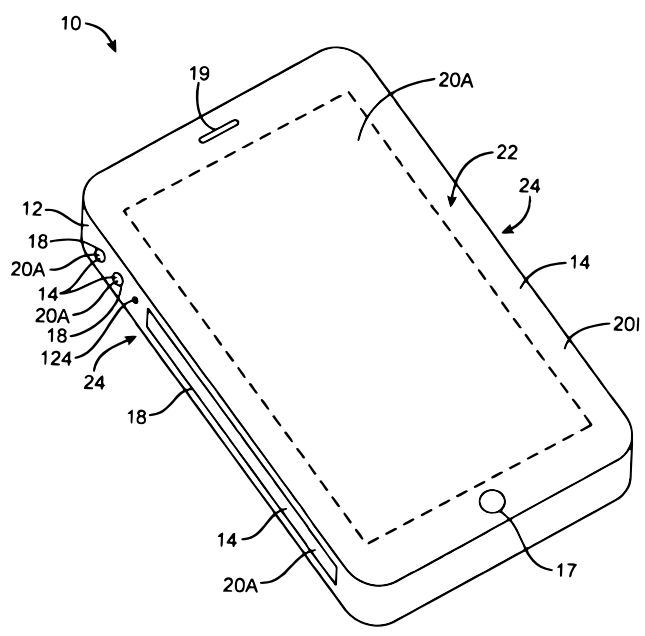

fragen die vorliegenden Texte danach, wie sich Zukunft in ein Denken übertragen lässt, das nicht in eurozentrische Teleologie eingebettet wäre.

Der Band Ethnofuturismen von Avanessian und Moalemi versucht in diesem Sinne nichts Geringeres, als «Freiheit und Emanzipation jenseits antiquierter Fortschrittslogik» (S.9) zu denken und kompiliert Theoriestücke und Spekulationen, die lokale Ausformungen pluraler Zukünfte ausfindig machen. Auch Lothian geht von einer solchen Vielzahl der Zukunftsfiktionen aus. Ihr Buch Old Futures. Speculative Fiction and Queer Possibility folgt einer queeren Tradition spekulativer literarischer und filmischer Erzählungen im 20. Jahrhundert. Lothian untersucht das Verhältnis dieser Texte zu einem Zukunftsdenken, das die Durchsetzung und den Erhalt imperialer Machtstrukturen und der Vormachtstellung einer technologischen Moderne sicherstellt. (S.2) Die Herausgeberinnen von We Travel the Space Ways. Black Imaginations, Fragments, and Diffractions, Gunkel und lynch, gruppieren wiederum kulturwissenschaftliche Kritik, aktivistische und künstlerische Beiträge um das Vorhaben eines Schwarzen future fictioning, das aus queerfeministischer Perspektive zu afrofuturistischen Spekulationen ansetzt. Dieses Einbiegen in die space ways bricht mit modernen Ordnungen von Zeitlichkeit, Subjektivität und Sinnstiftung und denkt Afrozukunft als Raum, in dem nicht-repressive Strukturen der Vergemeinschaftung und dekoloniale Wissensformationen erfahrbar werden.

Zukunftsdebatten scheinen, nicht zuletzt vor dem Hintergrund der ökologischen Sorge, Konjunktur zu haben. Die drei Publikationen greifen die aktuelle
Popularität afrofuturistischer Ästhetiken und Erzählungen auf und verhandeln die immer schon prekäre Ausgangslage marginalisierter Zukünfte vielleicht auch als ein Gegengewicht zum Krisendenken des Anthropozändiskurses, welcher, wie Kathryn Yusoff argumentiert, als geologische Wissenskategorie konstitutiv von rassisierten Machtstrukturen durchzogen ist. ${ }^{5}$ Doch stellt sich in Bezug auf den Erscheinungskontext der besprochenen Publikationen und die weiße Positionierung der Rezensentin ebenso die Frage nach dem Ort des Sprechens über marginalisierte Zukünfte: Was sind die machtstrukturellen Implikationen dieses Absolutsetzens der Positionierung einer queer person of color als kritische Instanz in dieser Rezension sowie innerhalb nordamerikanischer und europäischer Wissenschaftsdiskurse? Wie können Andere Zukünfte perspektiviert werden, ohne dass sie im Projekt der Inklusion marginalisierter sozialer Positionen aufgehen und wiederum einen weißen Zukunftsdiskurs zentrieren?

Der Band Ethnofuturismen führt Zukunft als ein Feld ein, das machtpolitischen Aushandlungen unterliegt und einer solchen Reflexion einen vielversprechenden Ausgangspunkt bieten würde. Doch zielen die Herausgeber in ihren einleitenden «Befunde[n] zu gemeinsamen und gegensätzlichen Zukünften» auf die Diagnose einer gegenwärtigen globalen Krise ab: Angesichts aufkommender Nationalismen in Europa und dem Niedergang der Nationalstaaten außerhalb Europas plädieren Avanessian/Moalemi dafür, in Diskussionen über mögliche Zukünfte globale Machtstrukturen und darin verzweigte Kapitalbewegungen einzubeziehen, wobei weltweite Ungleichheitsverhältnisse unterschiedlich gelagerte oder gar widersprüchliche Zukünfte hervorbringen. (S.9) Hinsichtlich der tagespolitischen Aktualität nimmt der Band zwangsläufig Abstriche in Kauf, handelt es sich bei den hier versammelten Beiträgen doch größtenteils um Erstübersetzungen bereits älterer Texte. Diese führen vor Augen, wie ständigem Wandel unterliegende regionale Bedingungen und kollektive Identitäten sowohl das Denken historischer Zeitlichkeit als auch spekulative Vorwegnahmen dessen, was kommen könnte, prägen. Insofern stellt Ethnofuturismen die Gültigkeitsansprüche futuristischen Denkens vor dem Hintergrund postkolonialer Debatten auf die Probe. Wie können ethnofuturistische Interventionen Spannungsverhältnisse zwischen allumfassenden Deutungsangeboten und partikularer Notwendigkeit emanzipatorisch wenden? Für die 
Herausgeber steht, obwohl sie dies einleitend ankündigen, nicht der Versuch im Zentrum, Gemeinsamkeiten und Gegensätzlichkeiten dieser Visionen unterschiedlicher historischer Prägung und spekulativer Priorisierung zu eruieren. Den titelgebenden Begriff der «Ethnofuturismen» entlehnen Avanessian/Moalemi einer estnischen literarischen Tradition zum Ende der Sowjetunion und legen ihn neu aus: als einen Ausweg aus der Dichotomie von "Multikulturalismus vs. Ethnopluralismus» und "Vision der Zukunft, die zwischen der Auflösung aller Unterschiede einerseits und der genau entgegengesetzten Ideologie einer Bewahrung von ursprünglichen Identitäten andererseits liegt». (S. و) In diesem Zusammenhang wäre eine kritische Einordnung des Begriffs des «Ethnischen` wünschenswert gewesen, der hier in pauschalisierender Geste dazu verwandt wird, eine Vielzahl Anderer Zukünfte zusammenzufassen, und damit auch die Dichotomie zwischen Partikularem und Universalem erneut aufzuspannen scheint.

Auf zwei konkurrierende Futurismus-Spielarten, die eines Schwarzen Futurismus und die eines Futurismus, der dem "Real-Fiktiv Hype» (S.15) untersteht - möchte ich etwas genauer eingehen. Hier zeigt sich, dass die Sammlung Ethnofuturismen einen «Widerstreit der Zukünfte» (S.9) mit jeweils divergierenden Ansätzen, gemeinsamen Linien und Streitpunkten auf den eigenen Seiten austrägt. Dies lässt sich zunächst an Aria Deans «Anmerkungen zur Blackzeleration» (S. 67-85) illustrieren: Dean unternimmt den Versuch, einen Akzelerationismus - also die Annahme, die Überwindung des Kapitalismus erfolge über die durch ihn bereitgestellten Mittel der Steigerung - abseits der Vereinnahmungen durch rechte und linke politische Lager von einer Schwarzen Position aus zu denken. Ausgehend von dieser «dritten Position` im Rassenkapitalismus, für die sich Subjektivität und Kapital gegenseitig konstituieren, bestätigt Dean den Akzelerationismus als integralen Bestandteil des Schwarzseins. (S. 80) Aus einer historischen Perspektive, in der «lebendige[s] Kapital, spekulative[r] Wert und akkumulierte Zeit [...] in den Körpern schwarzer, schon-inhumaner-(nicht-)Subjekte gespeichert sind" (S.85), gilt es, weder den Verlust der Humanität zu beklagen noch eine Wiederaneignung von Subjektivität voranzutreiben (S. 84). Damit zeichnet Dean die Grenzen nach, die den dominanten Zukunftsdiskurs der Moderne einfassen; dies gilt auch für Kodwo Eshuns 2003 verfasste und nun ins Deutsche übersetzte
"Weiterführende Überlegungen zum Afrofuturismus» (S.40-65) im selben Band. Vom unterschwelligen Verdacht bei Dean und Eshun, die 'Zukunft müsse aus nicht-eurozentristischer Perspektive bereits ausgedient haben, rücken die Texte von Steve Goodman und Anna Greenspan ab. Beide kommen aus dem Umfeld der Cybernetic Culture Research Unit, die von Nick Land - dessen Positionen Dean vehement kritisiert - in den 1990er Jahren an der Warwick University mitgegründet wurde. Indem sie sich dem Reiz des Spekulativen hingeben, legen sie weniger einen Gegenentwurf zu den von «Maskulinitäts- und Martialitätsphantasmen bestimmten westeuropäischen Futurismen des frühen 20. Jahrhunderts» (S.8) vor als spezifische Umformungen derselben und verlagern einen modernen Technologie- und Wissenschaftsglauben in neue regionale Kontexte: Goodmans Textcollage über die sinofuturistische Geheimwährung Fei Ch'ien (S. 87) erschließt das Spekulative als grundlegende kapitalistische Funktion. Anna Greenspans Überlegungen zum Retro-Futurismus auf der Weltausstellung in Schanghai 2010 zeichnen das Bild eines "absoluten Futurismus" (S.126), der unter dem Zugeständnis der Vorsilbe «retro〉 das Schanghai des 21. Jahrhunderts mit den Bildern des Futurismus aus dem 20. Jahrhundert bespielt. Diese real-fiktiven Zukunftsfigurationen verbleiben in engen Schleifen an das

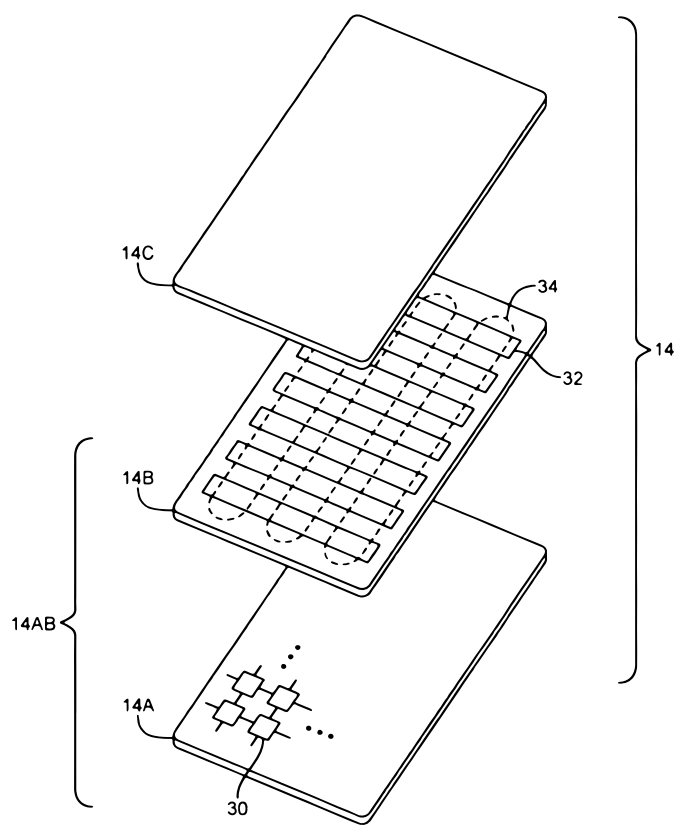


Archiv bekannter Zukünfte rückgekoppelt, die schließlich auch das Denken von Freiheit und Emanzipation einhegen. Bezüge auf die Zukunft als kolonisierte Trope und Verbund bereitstehender Deutungsmuster lassen die Anderen Zukünfte der Ethnofuturismen teils gar nicht so anders erscheinen.

Während der Band Ethnofuturismen einerseits von einem politischen Gegenwartsbezug ausgeht und zu den dabei aufgeworfenen dringlichen Fragen andererseits nur bedingt Antworten liefert, orientiert sich Alexis Lothian programmatisch am historischen Archiv der Zukünfte. (S.2) In Old Futures fächert die Autorin ein Spektrum queerer Zukünfte des 20. Jahrhunderts auf: angefangen bei spekulativen Feminismen des späten 19. und frühen 20. Jahrhunderts über Figurationen Schwarzer Zukünfte etwa bei W. E. B. Du Bois oder Samuel R. Delany bis zu filmischen Zukunftsspekulationen wie Lizzie Bordens Born in Flames (USA 1983) und Derek Jarmans Jubilee (GB 1978). Diese Zukünfte bewegen sich zwischen zwei Gravitationsfeldern: einer Zukunft als kolonisiertem Imaginationsraum der Moderne, deren Werden auf dem Ausschluss intersektional vergeschlechtlichter Personen des sogenannten globalen Südens beruht, und der Öffnung hin
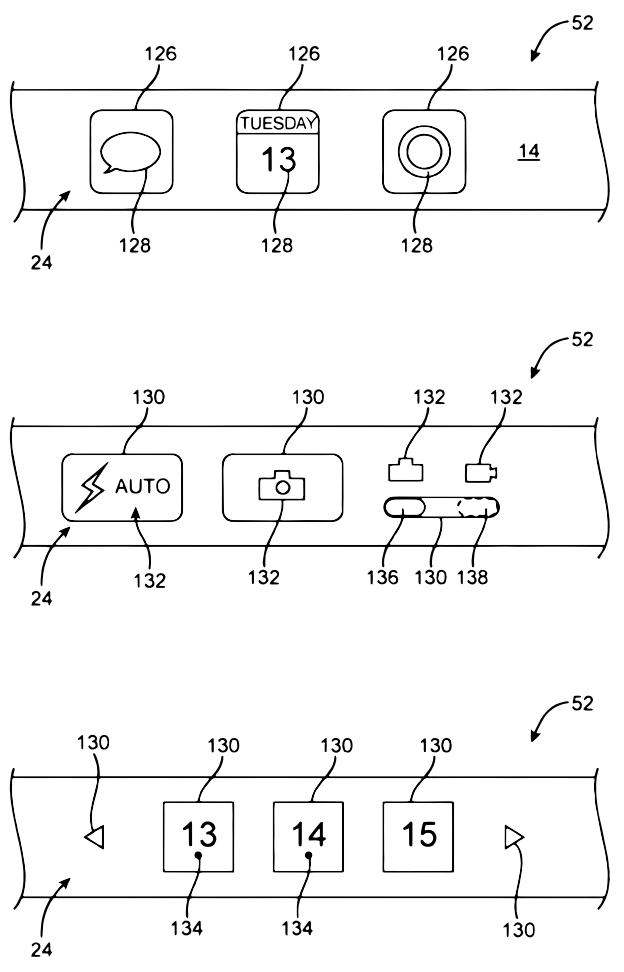

zu einer queeren Potenzialität, die zukünftige Sozialitäten in einem nicht-normativen Begehren der Gegenwart anlegt. (S. 29) In Erzählungen marginalisierter Zukünfte weist Lothian Schnittstellen mit queeren Rekonzeptualisierungen von Zeitlichkeit nach. Somit lässt Old Futures den bisher kaum erkundeten Nexus zwischen Theorien queerer Zeitlichkeit und den Modalitäten spekulativen Erzählens nachvollziehen. Der Begriff des world-making rekurriert hier sowohl auf einen fiktionalen Modus als auch auf ein zentrales Anliegen der Queer Studies (S.3), deren Theoriebildung Lothian genealogisch als \&Geschichte der Zukünfter versteht (S. 5). In diesem Rahmen legt Old Futures umfassende Analysen unterschiedlicher Kulturpolitiken des Spekulierens im 20. Jahrhundert vor. Zugleich tragen diese Diskussionen anschauliche Beispiele und Perspektiven in die Debatte um queere Zeitlichkeiten ein, die über die Alternativen von Lee Edelmans No-Future-Polemik und einer Zukunft als queerem Möglichkeitsraum wie bei Muñoz hinausweisen. ${ }^{6}$

Lothian befragt zunächst die spekulativen Fiktionen britischer und US-amerikanischer feministischer Autorinnen des frühen 20. Jahrhunderts wie z. B. Charlotte Perkins Gilmans Herland (1915) im Hinblick auf deren Verstrickungen mit kolonialen Narrativen und Bildwelten. Die Möglichkeitsbedingungen dieser Zukünfte beziehen queere Sozialität mit ein, perpetuieren jedoch zugleich rassistische Gewaltstrukturen bzw. sind in imperiale Projekte US-amerikanischer und europäischer Nationalismen eingelassen. (S.72) Die Annahme, dass sich Queerness und moderne Zukunft über soziale Kontexte hinweg gegenseitig ausschließen, erweist sich als nicht haltbar.

Dieser Befund bestätigt sich für Lothian in der Fokussierung ihrer Lektüren auf spekulative Fiktionen einer Schwarzen Tradition, die Entwürfe technologischer und reproduktiver Zukünftigkeit adaptiert und umdeutet. (S. 101) Die Autorin analysiert, wie afrofuturistische Figurationen alternativer Raumzeitlichkeit in die Artikulation von Zukunft als radikalem Möglichkeitsfeld einwirken. Ein «breeding futures» als queerem Reproduktionsmodus, wie Lothian mit Audre Lorde formuliert (S.100), schafft Weisen der Relationalität und der Gemeinschaft im lustvollen Selbstzweck queeren Begehrens. So liest Lothian Octavia Butlers Fledgling (2005) und Jewelle Gomez' The Gilda Stories (1991) als afrofeministische Vampirmythologien der Reproduktion über (deviante) Linien der Blutsverwandtschaft. (S. 116) 
Diese Lektüren verkomplizieren Positionen queerer Zeitlichkeit und Varianten sozialer Reproduktion, dennoch reicht der Beitrag, den Old Futures m. E. leistet, über das Feld der Kritik hinaus. Denn in den medialen Anordnungen der Rezeption audiovisueller Zukünfte erkennt die Autorin Möglichkeitsräume, mit denen ihre Argumentationslinie auf produktive Weise selbst in den Bereich des Spekulativen übersetzt. Mit dem Vermögen, Affekte durch Raum und Zeit zu übertragen, gesteht Lothian Bildschirmmedien eine eigene Qualität spekulativer Queerness zu. (S. 213) Dabei nimmt das Erproben verschiedener Medien und Modalitäten des Spekulierens selbst Raum im Buch ein. So bedient sich Old Futures des strukturellen Genre-Kniffs von 'Wurmlöchern', mithilfe derer sie historische und intermediale Querverbindungen zwischen den Old Futures und jüngeren Beispielen spekulativer Fiktionen zieht. Darüber hinaus berichtet die Autorin über ihre praktischen Erfahrungen als Teil des vidding-Fandoms. Die Fangemeinde der vidders eignet sich das Remixen von Videomaterial als reparative Praxis an, mit der sich hegemoniale audiovisuelle Zeitlichkeiten umordnen und die darin eingelassenen rassisierten und sexuierten Ordnungen umschichten lassen. (S.233) Lothians Auseinandersetzung mit alten Zukünften formuliert den Wunsch, eine Neuverteilung der Zukunft in der Gegenwart zu bewerkstelligen. (S. 22) Das vidding hält dafür die Mittel bereit.

Auch in We Travel the Space Ways tritt Afrofuturismus mehr als Tun und kreative Praxis denn als theoretisches Konstrukt in Erscheinung. Im Band kreisen die Bits unterschiedlicher medialer Figurationen und Ästhetiken des Spekulierens im Orbit einer Tradition Schwarzer visionärer Praktiken: Das Feld des black future visioning als Kulturkritik erweitern die Herausgeberinnen Henriette Gunkel und kara lynch um die Bereiche Fotografie, bildende Kunst, Architektur, Performance, Musik und aktivistisches Organisieren. Damit legt der Band eine Sammlung multimodaler Interventionen vor, die in kultureller und politischer Hinsicht Vorschläge zur Formation von Identität, Kollektivwerdung und neuen Daseinsformen in der Welt machen. (S. 21) So gehe es zum einen darum, Narrativierungen der Zukunft nicht der historischen Vereinnahmung durch Siedlerkolonisierung und Weißsein zu überlassen. (S. 25) Und doch sei dem Ersinnen radikal anderer Realitäten, auBerweltlicher Sphären und "safe(r) spaces» (S.27) ein

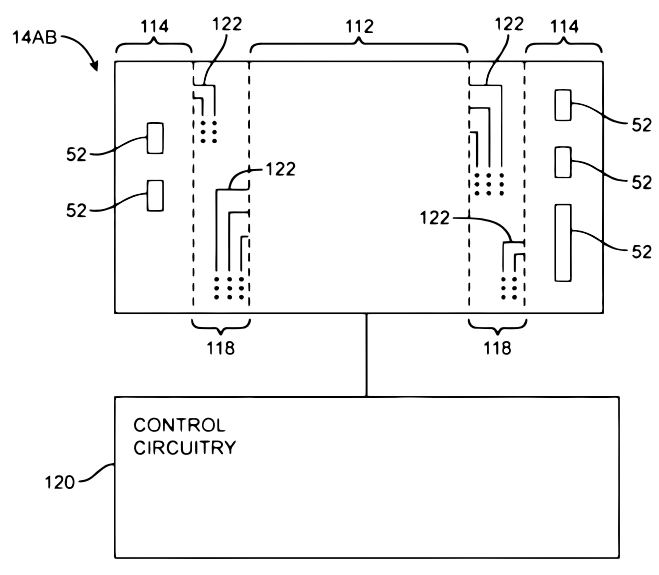

Bewusstsein um die Geschichte und Gegenwart intersektionaler Gewaltstrukturen eingeschrieben (S. 22).

Aus der gegenwärtigen Popularität afrofuturistischen Denkens leiten die Herausgeberinnen die Notwendigkeit $\mathrm{ab}$, die Historizität des Begriffs mitzudenken: Ein aktueller Afrofuturismus-Moment müsse in Anbetracht der historischen Dimension migrantischer Bewegungen sowie der kulturellen und technologischen Wissenstransfers über unterschiedliche geopolitische Kontexte hinweg immer neu befragt werden. (S. 23) We Travel the Space Ways weist dabei diskursiv und personell Berührungspunkte mit dem Afrofuturismus der 1990er Jahre auf, den Greg Tate im Band als «Rise of the Astro Blacks» rekapituliert. (S.199-202) Die queerfeministischen Positionierungen der Beiträge erlauben es jedoch, aus den gebahnten Wegen eines männlich und heteronormativ geprägten Diskurses auszuscheren. (S. 29) Zudem ist der Begriff des Afrofuturismus, wie die Herausgeberinnen anmerken, maßgeblich durch diasporische Akteur_innen geprägt. (S.24) Der Band bemüht sich daher, Beweggründe und historische Linien verschiedener "pan-African future projects" (S. 25) nachzureichen. Erprobt werden Strategien, um den Begriff der Zukunft aus dem Projekt der europäischen Aufklärung herauszulösen, das Afrika mit der Fähigkeit zur eigenen Geschichte auch eine Zukunft abspricht. Die Einbindung eines Konzepts der Africanicity destabilisiere etwa die im Humanismus verwurzelte binäre Trennung zwischen Nord und Süd in Analogie zur Differenz von (privilegiert) und sbenachteiligt). (S. 25) Diese space ways zu bereisen heißt, Zukunft als Fiktion, Metapher und Figur des Politischen zu begreifen, in der chromgestählte 


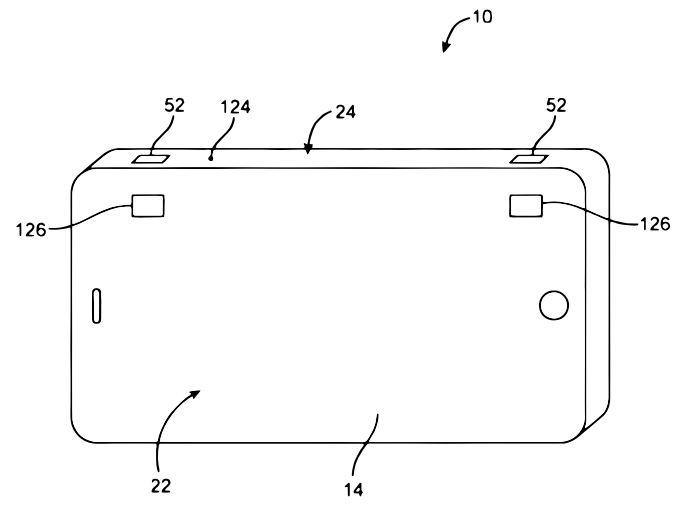

space-race-Fantasien und Dekolonialität einen Möglichkeitsraum teilen.

Einen Angelpunkt des konzeptuell und thematisch zwischen kreativem Schaffen, Aktivismus und Theoriekritik breit aufgestellten Bandes bilden Konzepte afrofuturistischer Zeitlichkeit, die Formen der Selbstwerdung und Vergemeinschaftung aus einer linearen zeitlichen Determiniertheit lösen. Die versammelten Modalitäten afrofuturistischer Zeitlichkeit sind eng mit Affekt- und Begehrensstrukturen verwoben und an situierte Politiken rückgebunden. (S.27) So überträgt die Aktivistin und Künstlerin Rasheedah Philipps in ihrem Beitrag den Agency-Impetus eines organize your own auf Fragen zeitlicher Selbstbestimmung (S. 237-244), während Alexis Pauline Gumbs' Schreiben über die temporale Bedingtheit von Liebe und Verlust eine Black Astrophysics entwirft. (S. 15-20) Tobias Nagls Auseinandersetzung mit dem Werk des afrodeutschen Künstlers Daniel Kojo Schrade verfolgt einen forensischen Ansatz, dem Materialschichten als zeitliche Überlagerungen gelten. (S. 321-342) Henriette Gunkel geht der Verschränkung von Afrofuturismus und queerer Zeitlichkeit in Filmbeispielen nach (S. 387-404), wobei die Performanz des (un)doing «devianter» Genderidentitäten ebenso normative Zeitordnungen unterwandert. Hier knüpft Kara Keelings Darstellung und Analyse eines dem Phänomen Disco inhärenten (im)proper sonic habitus an, der die Gendernormen in den USA der 1970er Jahre herausforderte und gleichzeitig die affektiven Verflechtungen Schwarzer Existenz, Geschichte, Technologie und Musik als visionären Modus ausweist, alternative soziale Konstellationen anzuleiten (S. 245-250, hier 245 f.) Die bei Keeling implizite und im Afrofuturismus zentrale Frage nach dem Verhältnis von race und Technologie beschäftigt Grisha Coleman und Thomas F. DeFrantz im Gespräch über ihre Medienkunst: Welche Technologien bringen die Anliegen Schwarzer Personen auf persönlicher, kollektiver und politischer Ebene voran? (S. 53-68)

In der multimodalen Aufstellung des Sammelbands betrifft die Frage nach Technologie auch jene Kulturtechniken, die Wissen generieren und bestimmen, worin dieses Wissen besteht. We Travel the Space Ways lotet aus, wie Schriftlichkeit als vorherrschende epistemische Praktik an ihre Grenzen gerät, indem weitere Wissenspraktiken in den geisteswissenschaftlichen Diskurs aufgenommen werden. Mit der Erweiterung der medialen Formate werden auch implizite Hierarchien zwischen den Arten und Weisen intellektueller und künstlerischer Diskursivierung verhandelt. Kiluanji Kia Hendas unter dem Titel A City Called Mirage versammelte skulpturale Arbeiten (S.47-52, siehe auch S. 287-302) z. B. setzen dem von Kolonialgeschichte und Unabhängigkeitskrieg geformten Raum der angolanischen Wüste neue Strukturen auf, während Ayesha Hameeds Performance-Skript ihrer Spekulationen über den Black-Atlantis-Mythos typografische Akzente setzt und Beschreibungen, Links zu Video-Clips sowie eingeblendete Folien beinhaltet. (S. 107-126) Zur Gestaltung einer Afrozukunft stellen die spekulativen Arrangements der einzelnen Beiträge je eigene produktive Dynamiken, Aushandlungs- und Erfahrungsräume bereit, die den Grundannahmen humanistischer Wissensproduktion mit alternativen Entwürfen begegnen.

Wie schon in Simones 22nd Century anklingt, erzählen diese Geschichten des 22. Jahrhunderts die Historien des 20. und vorheriger Jahrhunderte oftmals mit. Während das Projekt der Ethnofuturismen zu weiten Teilen darin besteht, tradierte Zukunftsfiktionen fortzuschreiben, bringen Old Futures und We Travel the Space Ways Chronopolitiken der medialen Anordnungen auf den Weg, die soziale Asymmetrien der Gegenwart justieren und zugleich Brüche in modernen Zukunftsdiskursen einleiten. Andere Zukünfte werden dabei nicht als kohärente Szenarien greifbar, sondern scheinen vielmehr im Möglichkeitsfeld spekulativen Mediendenkens auf: Von der Umstrukturierung audiovisueller Zeitlichkeit durch vidding-Fandoms bis zur Performance als intermedialer Konfiguration der Gegenerinnerung positionieren sich diese in der Vielstimmigkeit künstlerisch-medialer Praktiken und kollaborativer Arbeitsweisen und widmen 
hegemoniale Setzungen von Zukunft und Zeitlichkeit auf der Ebene ihres Ausdrucks um. Ein solches Tun vorausschauenden Zurückblickens nimmt unter afrozentrischen und intersektionalen Parametern Eintragungen in die Geschichte des 22. Jahrhunderts vor und verweist auf Konstellationen des Zukünftigen - als eines Vielfachen zwischen historisch-traumatischer Disposition und radikalem Umbruch.

1 José Esteban Muñoz: Cruising the Toilet. LeRoi Jones/Amiri Baraka, Radical Black Traditions, and Queer Futurity, in: GLQ: A Journal of Lesbian and Gay Studies. Bd. 13, Nr. 2-3 2007, 353-368, hier 364; Lee Edelman: No Future: Queer Theory and the Death Drive, Durham, 2004.

2 Lucian Hölscher: Die Entdeckung der Zukunft, Göttingen 2016.

3 Nina Simone: 22nd Century, auf: Here Comes the Sun, RCA Victor 1971. Simone coverte den Song von dem bahamaischen Musiker Exuma, der diesen im selben Jahr veröffentlichte.

4 Walter D. Mignolo: The Darker Side of Western Modernity. Global Futures, Decolonial Options, Durham 2011, 2.

5 Kathryn Yusoff: A Billion Black Anthropocenes or None, Minneapolis 2019, 4.

6 José Esteban Muñoz: Cruising Utopia. The Then and There of Queer Futurity, New York, 2007; Edelman 2004.
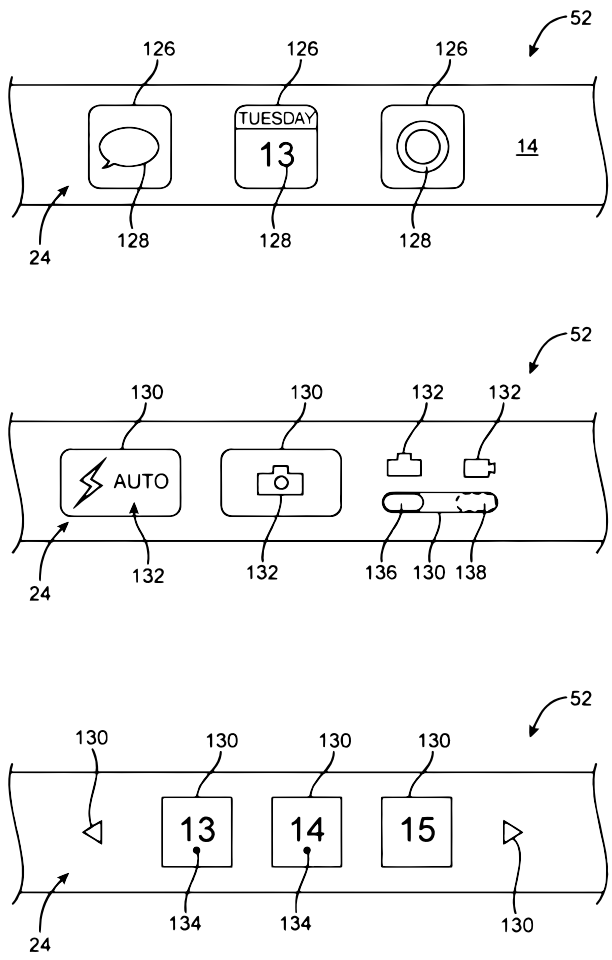